\title{
The APEX digital Fast Fourier Transform Spectrometer
}

\author{
B. Klein, S. D. Philipp, I. Krämer, C. Kasemann, R. Güsten, and K. M. Menten
}

Max-Planck-Institut für Radioastronomie (MPIfR), Auf dem Hügel 69, 53121 Bonn, Germany e-mail: [bklein; sphilipp; kraemer; kasemann; rguesten; kmenten] @MPIfR-Bonn.MPG.de

Received 11 April 2006 / Accepted 27 April 2006

\begin{abstract}
Context. We present the technology and first scientific results of a new generation of very flexible and sensitive spectrometers, wellsuited for the needs of spectral-line radio and (sub)millimeter astronomy: Fast Fourier Transform Spectrometers (FFTS), which are in operation at the Atacama Pathfinder EXperiment (APEX) telescope.

Aims. The FFTS for APEX is a novel high-resolution $2 \times 1 \mathrm{GHz}$ bandwidth digital spectrometer backend. Due to its high frequency resolution, and the demonstrated capability of operating at high altitude, the FFTS became the facility spectrometer for spectral line observations at APEX

Methods. The FFTS is based on one of the currently most powerful digitizer/analyzer boards available from Acqiris, Switzerland. The board incorporates two 1 Gsamples/s analog-to-digital converters (ADCs) with 8-bit resolution which feed an on-board complex field programmable gate array (FPGA) chip. The enormous processing power by today's FPGAs allow for a complete real-time FFT signal processing pipeline to decompose a $1 \mathrm{GHz}$ band into 16384 spectral channels in just one chip.

Results. Since May 2005 an MPIfR FFTS has been extensively used in all regular spectroscopic science observations. The performance at APEX was demonstrated to be very reliable and as good as measured in the first laboratory tests which finally led to the request to provide a second, facility type FFTS for APEX. The unit was delivered and commissioned in March this year.

Conclusions. Using a commercially available digitizer board, it was possible to develop a complete FFTS in only a few months. Successful observations at APEX demonstrate that this new generation of FPGA-based spectrometers easily matching and superseding the performance of older technology spectrometers and can built up much more easily. Furthermore, the by now available class of new high-speed ADCs and the continuous increase of FPGA processing power makes it very likely that FFTS can be pushed to broader bandwidth and even more spectral channels in the near future.
\end{abstract}

Key words. instrumentation: spectrographs - techniques: spectroscopic

\section{Introduction}

Spectroscopy allows studies of the physics and chemistry of astronomical objects such as planetary atmospheres, circumstellar envelopes and the interstellar medium of our Milky Way and external galaxies. Today, spectrographs offer usable contiguous bandwidth from a few $\mathrm{MHz}$ to $1-2 \mathrm{GHz}$, with a few thousand spectral channels capable of resolving narrow spectral lines e.g. of masers or thermal line emission of gaseous clouds. Particularly for the new APEX ${ }^{1}$ sub-millimeter telescope (Güsten et al. 2006) which operates at sky frequencies of several $100 \mathrm{GHz}$ up to $1.5 \mathrm{THz}$, a large bandwidth is very important to allow for studies of broader lines even at the highest frequencies.

The novel FPGA-based FFTSs offer new perspectives: Today, complex FPGAs have become very popular for building fast and reconfigurable hardware. State-of-the-art FPGAs include several hundreds of dedicated $18 \times 18$ bit multipliers which allow up to $8 \times 10^{10}$ multiplications and nearly $5 \times 10^{11} 36$-bit additions per second, giving these FPGA chips over $\sim 50$ times higher performance in digital signal processing than the latest Intel Pentium 4 processors. By parallelization of single processing threads and using the FFT algorithm in a pipelined manner

1 APEX is a collaboration between the Max-Planck-Institut für Radioastronomie, the European Southern Observatory, and the Onsala Space Observatory. it becomes possible to implement $16 \mathrm{~K}$-point Fourier transforms for input data rates up to 2 GBytes/s.

In this paper we describe the hardware and the FPGA signal processing of the FFTS developed for APEX. After a short review of common spectrometer techniques in radio astronomy (Sect. 2), we outline in Sect. 3 the technical design and the processing pipeline of the FFTS as well as the intermediate frequency (IF) processor at APEX. Sections 4 and 5 present the performance parameters and some selected spectra observed with the APEX telescope.

\section{Spectrometer for radio astronomy}

Since commercially available spectrum analyzers use frequency sweepers to obtain a broad-band spectrum, they are not sensitive enough for radio astronomical applications. Therefore, dedicated instruments have been developed for coherent spectral analysis. For radio frequencies one can identify three basic types of spectrometers: filter banks, acousto-optical spectrometer, and autocorrelators. Early on, spectrometers were build using analog (bandpass) filters and detectors, which were then assembled into filterbanks. Later, acousto-optical spectrometers (AOS) were developed which allowed for wider bandwidth and a larger number of spectral channels (Schieder et al. 2003). Due to their design they they need a stable, thermally controlled environment, which put constraints on their operability. The most commonly 


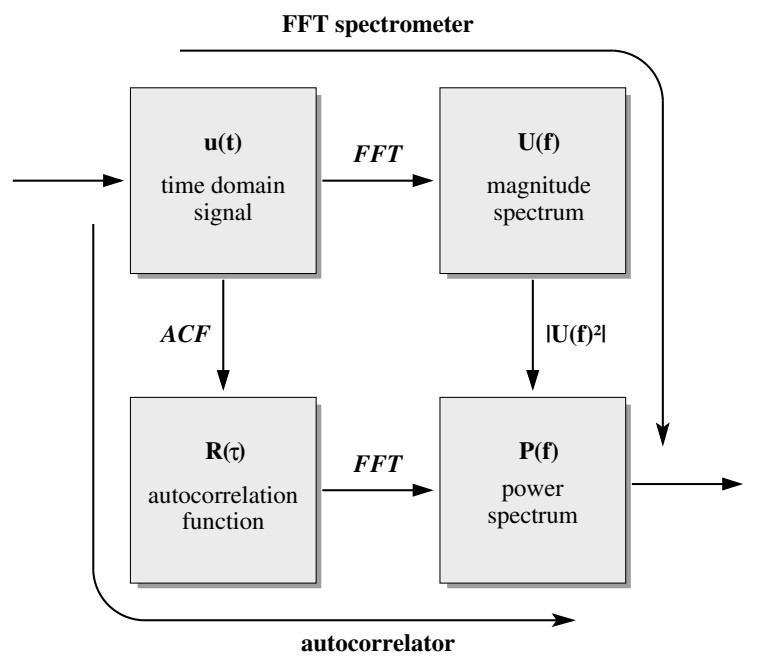

Fig. 1. Visualization of the "Wiener-Khinchin-Theorem".

used spectrometers are digital autocorrelators (AC). Their technology is based on the Wiener-Khinchin-Theorem, stating that the power spectrum of a signal is the Fourier transform of its autocorrelation function (ACF), as illustrated in Fig. 1. The advantage of this indirect method is the possibility to accumulate the ACF even before the Fourier transform produces the final power spectrum, which reduces the number of computations and enabled the calculation of the FFT in the early days. The ACF is computed by multiplication of the periodically time-shifted signals, followed by a summation of these. Limiting the sampler resolution to 1 or 2 bits, these three operations (shift, multiplication, addition) can be reduced to simple logical elements, and the ACF can be integrated in a customized silicon chip. This reduction to 1 or 2 bit (mostly 3-level) data sampling, however, results in a reduced sensitivity. Nevertheless, ACs have until recently been the only way to make possible the computation of CPU intensive Fourier transforms, so that bandwidths of order $1 \mathrm{GHz}$ were manageable.

\section{Development of the APEX FFTS}

The rapid increase in the sampling rate of commercially available analog-to-digital converters (ADCs) and the increasing power of field programmable gate arrays (FPGAs) chips has led to the possibility to directly digitize the down-converted intermediate frequency (IF) signals of coherent radio-receivers, and to transform the digital signal into a spectrum in real-time. Based on the technological progress and the experiences gained in building a narrow-band FFTS (Stanko et al. 2005) the APEX FFTS was developed by the MPIfR Digital Group.

\subsection{FFTS: hardware}

The APEX FFTS is based on the currently most powerful digitizer/analyzer board available from Acqiris, Switzerland. It incorporates two $1 \mathrm{GS} / \mathrm{s}$ ADCs which feed a XILINX Virtex 2 Pro70 FPGA chip. By combining both ADCs in an interleave manner (180 deg phase-shift), the board is capable to sample an analog input signal at $2 \mathrm{GHz}$ clock rate which results in a $1 \mathrm{GHz}$ Nyquist bandwidth. For a more detailed description of the FFTS hardware, especially the AC240 Board, we refer to Benz et al. (2005) and the Acqiris ${ }^{2}$ documentation.

\footnotetext{
2 www.acqiris.com
}

\subsection{FFTS: signal processing pipeline}

The digital signal conversion from the time domain to an integrated power spectrum is done in only one complex FPGA chip. The spectrometer core development was contracted to RF Engines Ltd. (RFEL), following our guidelines, and finally integrated at MPIfR. The core is based on RFEL's HyperSpeed Fast Fourier Transform (FFT) technology. It receives 8-bit samples from the two ADCs at a continuous sample rate of $2 \mathrm{GHz}$, and then processes this data in a sequence of four steps, as indicated in the flow of Fig. 2: first, a Digital Half Band Filter (DHBF) converts samples to a complex $I / Q$-format, and reduces the sample rate by a factor of two (decimation), which eases the subsequent processing requirements. A useful side effect of the DHBF is its ability to reduce the level on both bandpass sides. Indeed, this limits the useable bandwidth to $\sim 900 \mathrm{MHz}$ but has the great advantage of decreasing aliasing effects caused by real analog filters with finite stop band rejection. The DHBF is followed by the application of a windowing function, which weights the data in order to control the filtering performance of the FFT. The coefficients are user programmable at runtime, allowing the performance characteristics of the spectrometer to be modified for changing operational scenarios. For a high resolution and high dynamic-range spectrometer a 3-term Blackman-Harris window is a good compromise between sidelobe reduction, sidelobe fall-off and main lobe widening. For a comprehensive discussion about window functions and there properties, the interested reader can refer to Nuttall (1981). The 16 K-point HyperSpeed FFT core from RFEL forms the central element of the system, performing the conversion from the timedomain to the frequency-domain, and it includes bit reversing to sort the data in natural frequency order. The FFT is built using a highly parallel architecture in order to achieve the very high data rate of 2 GBytes/s. The final step of processing contains the conversion of the frequency spectrum to a power representation, and successive accumulation of these results. This accumulation step has the effect of averaging a number of power spectra, thereby reducing the background noise and improving the detection of weak signals. This step also reduces the huge amount of data produced by the earlier stages, and eases any subsequent interface bandwidth requirements and processing loads. The output from the spectrometer core is in a 32-bit floating-point format. With an ordinary PC for further data-managing and data transfer, the FFTS is integrated into the APEX control network and interfaces with the control system APECS via SCPI commands, as described in Muders et al. (2006).

\subsection{FFTS: frequency resolution}

To understand the spectral resolution and effective channel bandwidth of the FFTS, the spectral leakage caused by the application of the Fourier Transform (FFT) to a noisy signal must be considered. Spectral leakage is the result of the assumption in the FFT algorithm that the signals are contained in a single FFT time record, and thus periodic at intervals corresponding to the length of this time record. If the time record has a non-integral number of cycles, this assumption is violated and spectral leakage occurs, which introduces a wide range of frequencies in the frequency domain and in consequence the energy of a signal is spread to adjacent frequency bins (Harris 1978). If no window function is applied prior to the FFT, the first side lobes are attenuated by $13 \mathrm{~dB}$ relative to the main lobe and the side lobe fall-off rate is $6 \mathrm{~dB}$ per octave. Consequently, the selectivity of a bare FFT is poor, which results in a large amount of ripples in the 


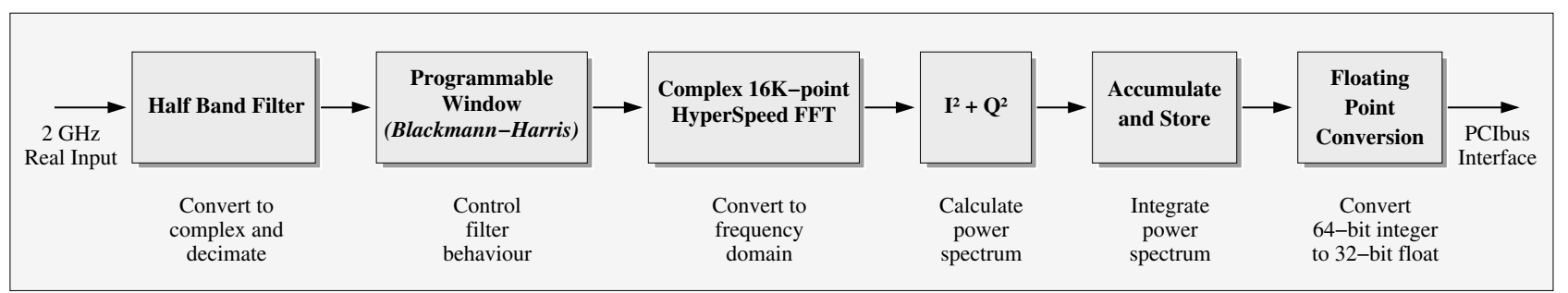

Fig. 2. A block diagram of RF Engines $2 \mathrm{GHz}$ Spectrometer Core for FPGA, as described in Sect. 3.2.

Table 1. Rectangular vs. Blackman-Harris window function.

\begin{tabular}{lcccc}
\hline \hline $\begin{array}{l}\text { Window } \\
\text { function }\end{array}$ & $\begin{array}{c}\text { Highest } \\
\text { side lobe }\end{array}$ & $\begin{array}{c}\text { Fall-off } \\
\text { rate }\end{array}$ & $\begin{array}{c}\text { 6 dB BW } \\
\text { (bins) }\end{array}$ & $\begin{array}{c}\text { ENBW } \\
\text { (bins) }\end{array}$ \\
\hline Rectangle & $-13 \mathrm{~dB}$ & $-6 \mathrm{~dB} /$ oct & 1.21 & 1.00 \\
Blackman-Harris & $-61 \mathrm{~dB}$ & $-6 \mathrm{~dB} /$ oct & 2.19 & 1.61 \\
\hline
\end{tabular}

passband. In addition, the spectral leakage from a large signal component may be so severe that other weaker signals at different frequencies are masked. The effects of spectral leakage can be restricted by reducing the discontinuities at both ends of the time record, i.e. by multiplying the data with a suitable window function. For radioastronomical applications a Blackman-Harris window is adequate due to its excellent spectral leakage attenuation and good amplitude conservation for random and noisy input signals. The APEX FFTS applies a 3-term Blackman-Harris window ${ }^{3}$. Unfortunately, there is always a trade-off between the main lobe width and the side lobe leakage: as the side lobe level decreases, the main lobe width is increased. To characterize this behaviour the equivalent noise bandwidth (ENBW) can be used. The ENBW indicates the equivalent rectangular bandwidth of a filter with the same peak gain that would result in the same output noise power. Table 1 is a comparison of the properties of a rectangular and a Blackman-Harris window. Since the ENBW for a rectangle filter is 1.0, which is equal to the channel separation frequency $(61.035 \mathrm{kHz})$, the frequency resolution for the APEX FFTS, applying the Blackman-Harris window, is $98.267 \mathrm{kHz}$.

\subsection{FFTS: IF processor}

Interfacing to the FFTS, an IF processor has been developed which is capable of down-converting the APEX-IF $(4-8 \mathrm{GHz})$ to baseband $(0-1 \mathrm{GHz})$. The system contains two identical IF chains for simultaneous observations with two receivers. An input multiplexer allows to connect each of the IF chains to six receiver outputs, providing the opportunity for fast switching between different receivers. In addition, it is feasible to connect one receiver to both FFTS with different IF offsets, thus providing effective bandwidths broader than $1 \mathrm{GHz}$ and up to $2 \mathrm{GHz}$, depending on the overlap area (see Sect. 5). To ensure an optimal input to the FFTS an autoleveling algorithm is implemented in the IF processor. The complete system is both, fully manually and remote controllable using the SCPI command interface (Muders et al. 2006).

\section{Specification and performance}

In Tables 2 and 3 we compile the specifications and the actually achieved performance of the APEX FFTS, its ADC/FPGA

\footnotetext{
${ }^{3} w(n)=0.5-0.49 \cos (2 \pi n / N)+0.0568 \cos (4 \pi n / N)$.
}

Table 2. Hardware specifications of the ADC/FPGA board AC240.

\begin{tabular}{lr}
\hline \hline ADC input sample rate & max 2 GS/s \\
ADC input bandwidth (-3 dB) & $0-1 \mathrm{GHz}$ \\
ADC input resolution (quantisation) & $8 \mathrm{Bit}$ \\
Maximum dynamic range (linear) & $48 \mathrm{~dB}$ \\
Input full scale range, selectable & $-22 \mathrm{~dB}-16 \mathrm{~dB}$ \\
Ghost free dynamic range & $>30 \mathrm{~dB}$ \\
FPGA Data Processing Unit & XILINX Virtex 2 Pro 70 \\
PCIbus interface & 32-bit, 33 MHz \\
\hline
\end{tabular}

Table 3. Specifications of the FPGA/FFT-processing pipeline.

\begin{tabular}{lr}
\hline \hline Fast Fourier Transform (FFT) & RFEL, HyperSpeed FFT \\
FFT - number of frequency channels & 16383 \\
FFT - channel separation & $61.035 \mathrm{kHz}$ \\
FFT - Blackman-Harris window & 3 term, $-61 \mathrm{~dB}$ side-lobe \\
FFT - frequency resolution & $98.267 \mathrm{kHz}$ \\
FFT - arithmetic & 2 's complement, $18-\mathrm{bit}$ \\
Digital Half Band Filter (DHBF) & $50-950 \mathrm{MHz}$ \\
DHBF - Stopband rejection & $+48 \mathrm{~dB}$ \\
DHBF - Passband ripple & max $\pm 0.1 \mathrm{~dB}$ \\
Power spectra builder (sum of squares) & full precision, 33-bit \\
On-board accumulation (integration) & $54-$ bit precision \\
Integration - max time (full precision) & $\sim 35 \mathrm{~s}$ \\
Integration - min time & $32.768 \mu \mathrm{s}$ \\
Stability/Allan-Variance: only FFTS & $\sim 4000 \mathrm{~s}$ \\
Stability/Allan-Variance: IF + FFTS & $\sim 500 \mathrm{~s} \mathrm{(Fig.} \mathrm{4)}$ \\
\hline
\end{tabular}

board and the FPGA/FFT-processing pipeline. To illustrate the performance we plot in Figs. 3 and 4 the results of stability tests, performed pre-shipment at MPIfR and after installation at the telescope.

Figure 3 presents the continuous decrease of the accumulated noise vs. integration time, which followed the radiometer formula, i.e. was $1 / \sqrt{t_{\text {int }}}$, for nearly two days of operation. Figure 4 displays an Allan-Variance plot of the spectrometer (incl. now the IF unit) as operated at the telescope, emphasizing the remarkable stability of both, the APEX IF and FFTS, on a timescale of $\sim 500 \mathrm{~s}$

\section{Observations}

Observations at higher frequencies, especially when operating at $\mathrm{THz}$ frequencies, require that spectrometers provide a larger bandwidth to allow for a complete coverage of the full line width of the observed object in one exposure. In Fig. 5 we show the spectra of the warm carbon monoxide towards the starburst nucleus of the galaxy NGC 253 as observed with APEX. Clearly, with an actual line width of more then $300 \mathrm{~km} \mathrm{~s}^{-1}$, a spectrometer bandwidth of only $1 \mathrm{GHz}$ is insufficient to study the emission of $\mathrm{CO}(7-6)$ at $806 \mathrm{GHz}$. However, by combining two $1 \mathrm{GHz}$ wide spectrometers, offset in their IF center frequencies, it was 


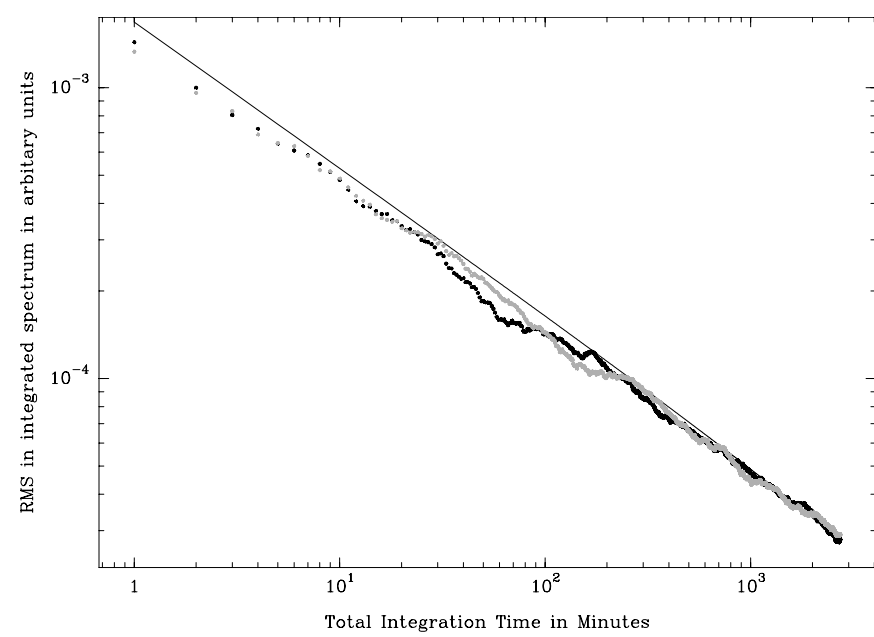

Fig. 3. Decrease of noise in accumulated spectra (60 s/dump) vs. total integration time, as measured with a noise diode. Both IF/FFTS channels are plotted (in black and grey), for comparison the expected slope $1 / \sqrt{t_{\text {int }}}$ is given.

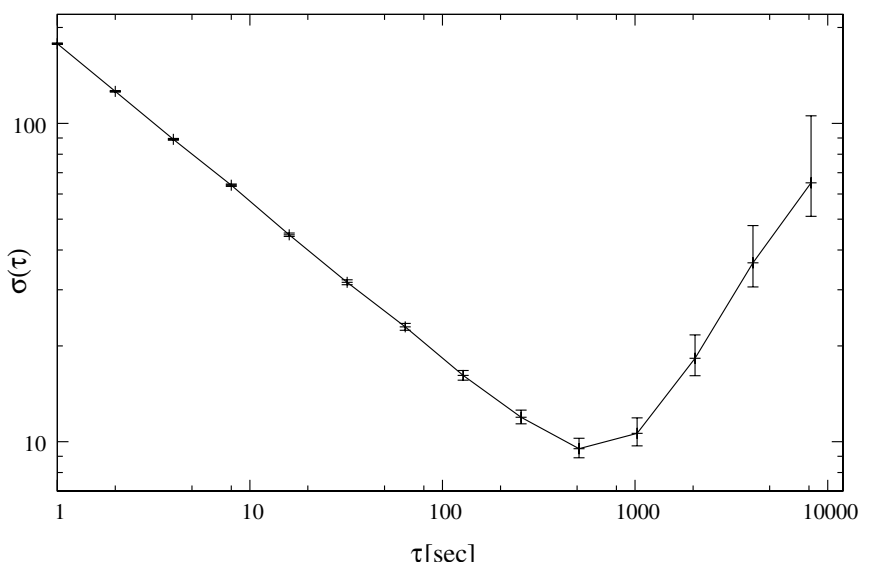

Fig. 4. The remarkable stability of the combined APEX FFTS/IF system is illustrated in this Allan-Variance-Plot. At the telescope, thus being exposed to all environmental disturbances, the spectrum of a noise source (5-7 GHz) was integrated and processed in the APEX FFTS/IF System. The spectroscopic variance between two $1 \mathrm{MHz}$ broad channels, separated by $600 \mathrm{MHz}$ within the band, was determined to be stable on a timescale of $\sim 500 \mathrm{~s}$.

possible to observe the complete emission in one integration, thus avoiding problems of cross-calibration, pointing errors, and platforming effects of otherwise sequential observations. On the other hand, to have a backend for spectral line surveys or narrow line sources, a high spectral resolution is required. The large number of spectral channels achievable with FFT spectrometers eliminates the disadvantage and complexity of having to reduce the bandwidth to get higher spectral resolution, which is commonly needed in autocorrelator systems, therefore making it better suited for combined broad band and high resolution observations.

The scientific results obtained during the first year of APEX operation as well as during telescope commissioning are presented in this A\&A volume.

\section{Conclusions and outlook}

The FFTS has been in routine operation since May 2005 at the APEX. The backend has been proven extremely reliable and

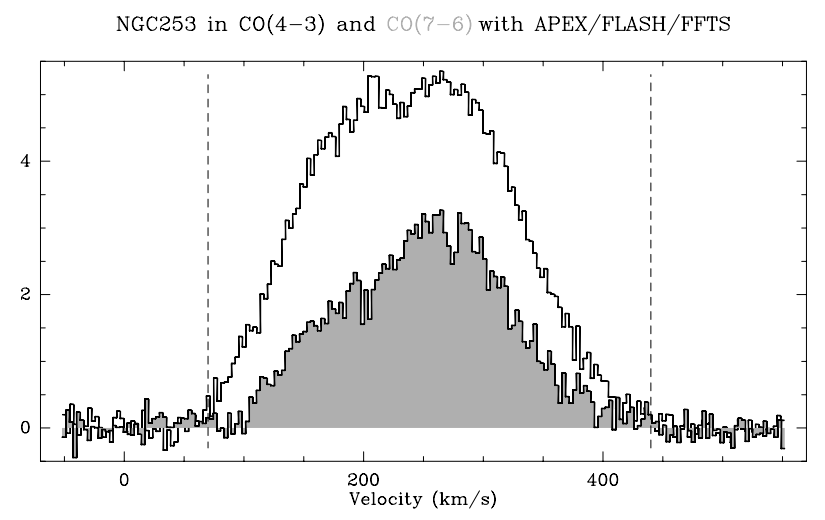

Fig. 5. Spectra of warm carbon monoxide towards the nucleus of NGC 253, observed with the FLASH receiver at APEX (Heyminck et al. 2006). The emission of $\mathrm{CO}(4-3)$ (at $461 \mathrm{GHz}$, top line), observed with $1 \mathrm{GHz}$ bandwidth, is superimposed on the $\mathrm{CO}(7-6)$ line $(806 \mathrm{GHz})$. The latter has been observed with an effective bandwidth of $1.8 \mathrm{GHz}$ (composed of two $1 \mathrm{GHz}$ bands, offset by $800 \mathrm{MHz}$ ). The dashed vertical lines indicate the bandwidth of $1 \mathrm{GHz}$ for the $\mathrm{CO}(7-6)$ line. Clearly, the velocity width is barely sufficient to define the zero level (baseline), emphasizing the need for wide bandwidth spectrometers for extragalactic work at submm frequencies.

robust, even under the harsh environmental conditions at this remote facility. The potential advantages of FPGA-based FFT spectrometer can be summarized:

- High bandwidth (1 to a few $\mathrm{GHz}$ in the future) with up to $16 \mathrm{~K}$ spectral channels $(32 \mathrm{~K}$ channels in preparation at MPIfR) in a single FPGA chip.

- Full signal sampling with 8-bit, no additional calibration by means of implicit total power measurements, thus higher sensitivity and stability in comparison with autocorrelators and acousto-optical spectrometers. In addition, this multi-bit sampling provides a higher dynamic input-range, so a less sophisticated autogain control is needed. Consequently, the IF processor is less complex, making the system more stable.

- Very high stability via pure digital signal processing.

- Modular design with calibration-free digitizer/analyzer boards, thus simple reproducibility.

- Low space and power requirements, thus safe to use at high altitude (e.g. APEX at $5100 \mathrm{~m}$ ) or well as on satellites.

- Low price compared to traditional spectrometers through use of commercial parts.

Our novel FPGA-based FFTS development offers exciting perspectives for high-resolution broadband spectrometers. The new class of high-speed ADCs and the advantages of FPGA, with their wide availability, decreasing costs (both based on the large commercial interest) and increasing processing capability (Moore's Law), makes it very likely that FFTS can be pushed to broader bandwidths in the near future.

\section{References}

Benz, A. O., Grigis, P. C., Hungerbühler, V., et al. 2005, A\&A, 442, 767

Güsten, R., Nyman, L.-Å., Schilke, P., et al. 2006, A\&A, 454, L13

Harris, F. J. 1978, Proc. IEEE, 66, 51

Heyminck, S., Kasemann, C., Güsten, R., de Lange, G., \& Graf, U. U. 2006, A\&A, 454, L21

Muders, D., Hafok, H., Wyrowski, F., et al. 2006, 454, L25

Nuttall, A. H. 1981, IEEE Trans. ASSP, 29, 84

Schieder, R. T., Siebertz, O., Gal, C., et al. 2003, in Proc. SPIE 4855, ed. T. G. Phillips, \& J. Zmuidzinas, 290

Stanko, S., Klein, B., \& Kerp, J. 2005, A\&A, 436, 391 